\title{
Expression of the RNase III enzyme DROSHA is reduced during progression of human cutaneous melanoma
}

\author{
Seyed Mehdi Jafarnejad ${ }^{1}$, Cecilia Sjoestroemํ․ Magdalena Martinka ${ }^{2}$ and Gang $\mathrm{Li}^{1}$ \\ ${ }^{1}$ Department of Dermatology and Skin Science, University of British Columbia, Vancouver, BC, Canada and \\ ${ }^{2}$ Department of Pathology, Vancouver Coastal Health Research Institute, University of British Columbia, \\ Vancouver, BC, Canada
}

\begin{abstract}
Aberrant expression of microRNAs (miRNAs) and their biogenesis factors has been frequently observed in different types of cancer. We recently reported that expression of DICER1 is reduced in metastatic melanoma. Nevertheless, so far very little is known about the expression pattern of other miRNA biogenesis factors in this type of malignancy. Here, we investigated the expression pattern of DROSHA in a large set of melanocytic lesions $(n=409)$ by tissue microarray and immunohistochemistry. We found that nuclear expression of DROSHA is markedly reduced in the early stages of melanoma progression $(P=0.0001)$ and is inversely correlated with melanoma thickness $(P=\mathbf{0 . 0 0 0 1})$, AJCC stages $(P=0.0001)$, and ulceration status $(P=0.002)$. We also confirmed the reduced expression of nuclear DROSHA by a second specific antibody raised against a different region of the DROSHA protein. In addition, we observed that the reduced nuclear expression of DROSHA during melanoma progression is accompanied by an increased cytoplasmic expression of this protein $(P=0.0001)$. Finally, we found that expression pattern of DROSHA varies from that of DICER1 and concomitant loss of expression of both DICER1 and DROSHA confers the worse outcome for melanoma patients. Our results demonstrate a reduced nuclear expression of DROSHA, which further highlights a perturbed miRNA biogenesis pathway in melanoma. In addition, the aberrant subcellular localization of DROSHA indicates possible deregulation in the mechanisms responsible for its proper localization in the nucleus.

Modern Pathology (2013) 26, 902-910; doi:10.1038/modpathol.2012.225; published online 1 February 2013
\end{abstract}

Keywords: DROSHA; melanoma; TMA; tumor progression

MicroRNAs (miRNAs) are a large family of singlestranded noncoding RNAs of $\sim 22$-nucleotides involved in posttranscriptional regulation of gene expression. Human genome produces more than a thousand miRNAs, each of which act via the RNA silencing pathway to regulate stability, degradation or rate of translation of the target mRNAs. ${ }^{1}$ With some exceptions, miRNAs are transcribed by RNA polymerase II from independent genes or as parts of introns of protein-coding genes in the form of pri-miRNAs. ${ }^{2}$ The pri-miRNAs are processed within the nucleus by microprocessor complex, core components of which are the RNase III enzyme DROSHA and the DiGeorge syndrome critical region gene 8 (DGCR8) proteins. The

Correspondence: Dr G Li, MD, PhD, Department of Dermatology and Skin Science, University of British Columbia, Research Pavilion, 828 W. 10th Avenue, Vancouver, BC, Canada V5Z 1L8. E-mail: gangli@mail.ubc.ca

Received 25 July 2012; revised 8 November 2012; accepted 14 November 2012; published online 1 February 2013 product of the microprocessor complex are $\sim 70$-nucleotide pre-miRNAs, which are subsequently transported to the cytoplasm by Ran GTPase-Exportin-5 complex. ${ }^{1}$ Upon arrival in cytoplasm, DICER1 further processes the premiRNAs to $\sim 22$-nt duplexes. One strand of each duplex, representing a mature miRNA, is then incorporated into the RNA-induced silencing complex (RISC), which then causes translational repression or deadenylation and degradation of the target mRNAs identified by base-pairing to the core sequences of the corresponding miRNAs. ${ }^{1}$

The known miRNAs' targets in human transcriptom are involved in a wide range of cellular functions such as development, ${ }^{3}$ proliferation and apoptosis $^{4}$ and angiogenesis. ${ }^{5}$ Therefore, dysregulation of miRNA biogenesis, hence alteration in miRNA expression levels, can lead to a variety of diseases, including cancer. Widespread aberrant expression of miRNAs has been frequently observed in cancers. ${ }^{6-8}$ These widespread changes in expression pattern of miRNAs could be due to 
chromosomal abnormalities ${ }^{9}$ or epigenetic effects and aberrant transcription. ${ }^{10,11}$ However, the aberrant expression or function of miRNA biogenesis factors could also potentially alter the miRNAome of the cells; therefore, contribute to the tumorigenesis process. Accordingly, expression of several miRNA biogenesis factors such as DICER1 and Argonauts have been observed to be deregulated in cancers. ${ }^{12-14}$

Widespread deregulated expression of miRNAs in melanoma has been observed in different studies ${ }^{15,16}$ accompanied by revelation of involvement of several individual miRNAs in regulation of different features of melanoma such as invasion, proliferation and evasion of apoptosis. ${ }^{16-18}$ We recently revealed that expression of DICER1 is reduced in metastatic melanoma, which was inversely correlated with survival of melanoma patients. ${ }^{19}$ Nevertheless, so far very little is known about the expression pattern of other miRNA biogenesis factors in melanoma. Several lines of evidence indicate that expression of DROSHA is deregulated in malignancies and may influence clinical outcomes. ${ }^{13,20-22}$ In this study, to identify the expression pattern of DROSHA in melanoma, we investigated the expression of its protein in a large set of melanocytic lesions by immunohistochemistry.

\section{Materials and methods}

\section{Construction of TMA}

All specimens were obtained from the 1990 to 1998 archives of the Department of Pathology, Vancouver General Hospital. The use of human skin tissues in this study was approved by the Clinical Research Ethics Board of The University of British Columbia. The most representative tumor area from each sample was carefully selected by a certified dermatopathologist and marked on the H\&E-stained slide. The TMAs were assembled using a tissue-array instrument (Beecher Instruments, Silver Spring, MD, USA). Duplicate $0.6-\mathrm{mm}$ thick formalinfixed paraffin-embedded tissue cores were taken from each biopsy specimen and spotted on five high-density TMA blocks. Four- $\mu$ m sections were cut with a Leica microtome and transferred to adhesive coated slides. Tissues from 29 normal nevi, 43 dysplastic nevi, 226 primary melanomas, and 111 metastatic melanomas were used in construction of the tissue microarray. In this study we also used a smaller TMA construct (two slides) with 48 dysplastic nevi, 86 primary melanomas, and 49 metastatic melanomas.

\section{Immunohistochemistry}

TMA slides or single tumor slides were dewaxed at $55^{\circ} \mathrm{C}$ for $30 \mathrm{~min}$ and three consequent washes with xylene. Tissues were rehydrated by a series of washes in 100, 95, and $80 \%$ ethanol, followed by two washes in distilled water. Antigen retrieval was done by heating the samples at $95^{\circ} \mathrm{C}$ for $30 \mathrm{~min}$ in $10 \mathrm{mmol} / \mathrm{l}$ sodium citrate (pH 6.0). After inactivating the endogenous peroxidase by incubating in $3 \%$ $\mathrm{H}_{2} \mathrm{O}_{2}$ for 30 min and blocking with universal blocking serum for $30 \mathrm{~min}$, slides were incubated with polyclonal rabbit anti-DROSHA antibodies (1:50 dilution, ab12286 Abcam, Cambridge, MA, USA; or 1:50; sc-33778 Santa Cruz Biotechnology, Santa Cruz, CA, USA) at $4{ }^{\circ} \mathrm{C}$ overnight. The slides were then incubated with biotin-labeled secondary antibody and streptavidin-peroxidase for $30 \mathrm{~min}$ each, followed by developing with diaminobenzidine substrate kit (Vector Laboratories, Burlington, ON, Canada) and counterstained with hematoxylin.

\section{Evaluation of Immunostaining}

The evaluation of DROSHA expression was made blindly by two independent observers (including one dermatopathologist) simultaneously. The nuclear or cytoplasmic DROSHA staining was scored into four grades according to the following staining intensities: $0,1+, 2+$, and $3+$. Percentages of DROSHA-positive cells were also scored into five categories: $0(0 \%), 1(1-25 \%), 2(26-50 \%), 3$ $(51-75 \%)$, and $4(76-100 \%)$. The immunoreactive score, which is calculated by multiplying the scores of staining intensity and the percentage of positive cells, was used as the final staining score.

\section{Statistical Analyses}

The Kruskal-Wallis test was applied to compare the DROSHA staining between normal nevi, dysplastic nevi, primary melanomas and metastatic melanomas using the GraphPad prism5 software. Other statistical analyses were performed with the SPSS 11.5 software. The correlations between DROSHA expression and clinicopathologic variables, including AJCC stages, age, gender, tumor thickness, location, and ulceration were analyzed by $\chi^{2}$ test. Spearman test was used to analyze the correlation between nuclear DROSHA and cytoplasmic DICER1 expression and correlation between nuclear and cytoplasmic DROSHA expression. In all cases, a $P$ value of $<0.05$ was considered significant.

\section{Results}

\section{Reduced Nuclear DROSHA Staining Correlates with Melanoma Progression}

We used a polyclonal rabbit antibody raised against the N-terminal (residues 1-100) of human DROSHA protein to investigate its expression pattern in 409 melanocytic lesions (29 normal nevi, 43 dysplastic nevi, 226 primary melanomas, and 111 metastatic 
melanomas). We observed a predominant nuclear DROSHA staining in different samples (Figure 1a). We also detected some cytoplasmic signal with this antibody in melanocytic lesions in all stages. A significant difference in nuclear DROSHA staining was observed between different stages of melanoma. Kruskal-Wallis test revealed a clear reduction in the expression of nuclear DROSHA during melanoma progression $(P=0.0001$; Figure 1b). We found significant reduction in expression of nuclear DROSHA from normal nevi to dysplastic nevi $(P=0.002)$ and from dysplastic nevi to primary melanomas $(P=0.0001)$ but not between primary melanomas and metastatic melanomas $(P=0.052)$. Similarly, when we divided the samples from each stage into two groups based on expression of nuclear DROSHA, we observed an increase in percentage of samples with no nuclear DROSHA staining during melanoma progression $(P=0.0001$; Figure 1c). Accordingly, while $82.7 \%$ of normal nevi and $62.7 \%$ of dysplastic nevi had positive nuclear staining for DROSHA, only $26.1 \%$ of primary melanomas and $17.1 \%$ of metastatic melanomas stained positive for nuclear DROSHA.

\section{Inverse Correlation between Nuclear DROSHA Staining and Tumor Thickness, AJCC Staging and Ulceration Status}

To assess whether reduced nuclear DROSHA staining correlates with clinicopathologic variables of the patients, we examined the expression pattern of nuclear DROSHA in 226 primary melanoma samples a
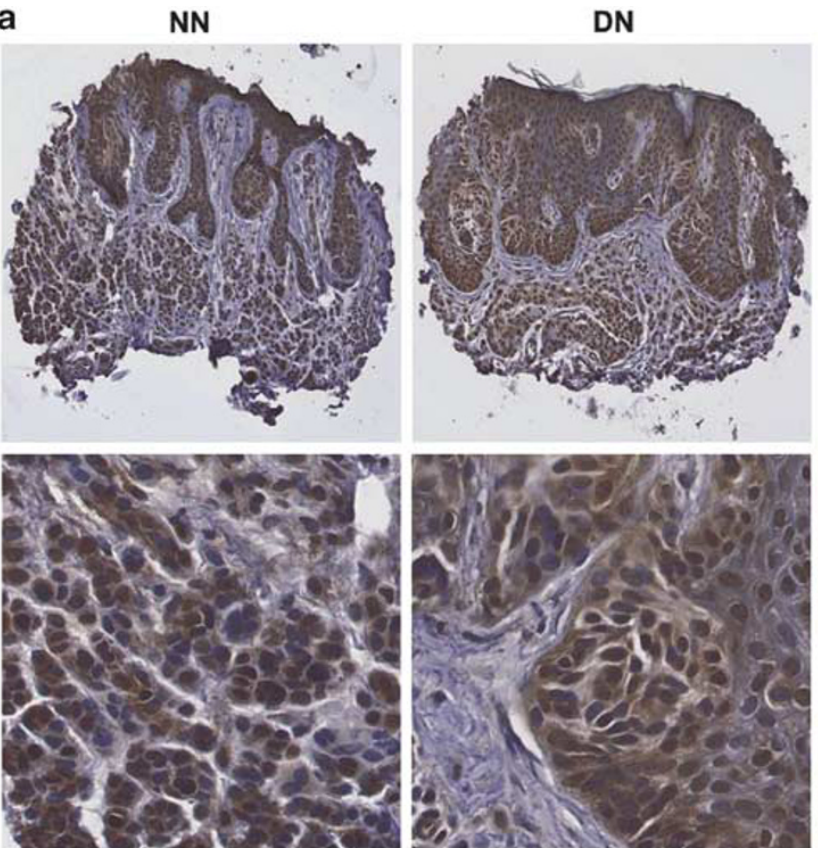

PM
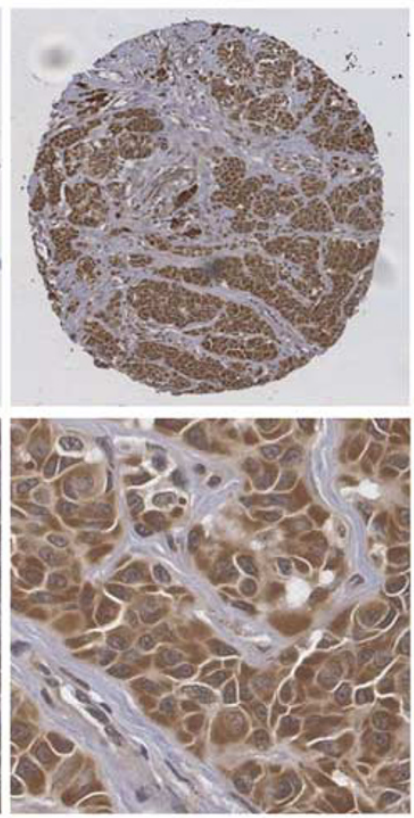
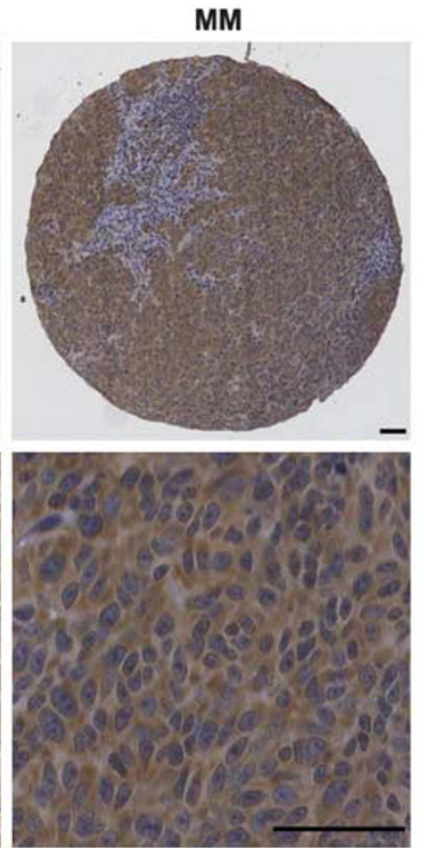

b

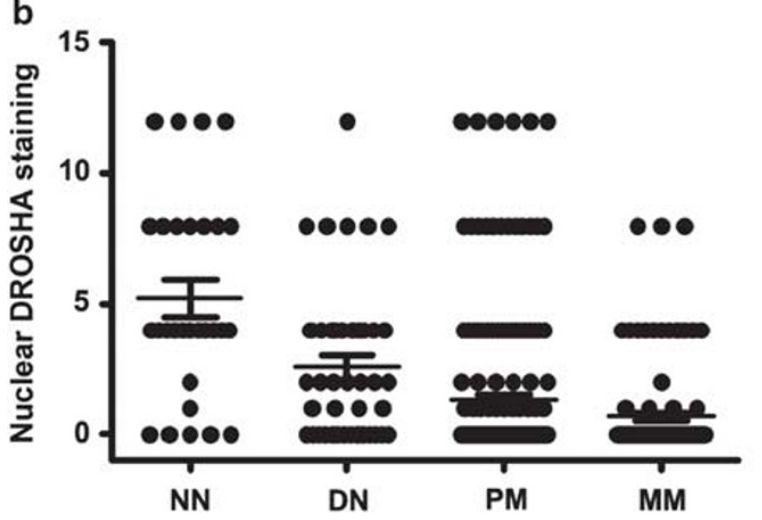

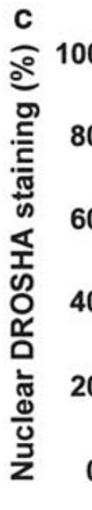

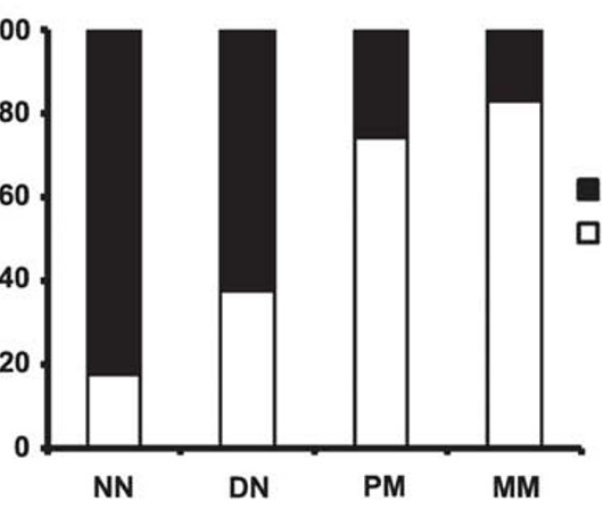

Positive (1-12)

$\square$ Negative (0)

Figure 1 Reduced expression of nuclear Drosha correlates with melanoma progression. (a) Representative images of normal nevi (NN) and dysplastic nevi (DN) with strong nuclear Drosha staining, primary melanoma (PM) with weak staining, and metastatic melanoma (MM) with negative nuclear Drosha staining. Scale bar $=50 \mu \mathrm{m}$. (b) Kruskal-Wallis test for differences in nuclear Drosha staining among NN, DN, PM, and MM. Median is depicted as a horizontal line in each group $(P=0.0001)$. (c) $\chi^{2}$ test for differences in nuclear Drosha staining in NN, DN, PM, and MM. Significant difference was found between DN and PM $(P=0.0001)$ but not NN and DN $(P=0.068)$ or $\mathrm{PM}$ and $\mathrm{MM}(P=0.066)$. 
(Table 1). Although DROSHA staining did not have a significant correlation with patients' age or sex, location of tumor and lymphocytic response, it showed a significant inverse correlation with tumor thickness (Breslow's depth of invasion). Accordingly, percentage of samples with positive staining for nuclear DROSHA reduced from $41.0 \%$ in tumors $\leq 1 \mathrm{~mm}$ thick to $18.2 \%$ in tumors thicker than $1 \mathrm{~mm}$ $\left(P=0.0001, \chi^{2}\right.$ test; Figure 2a). We also observed an inverse correlation between expression of nuclear DROSHA and ulceration status of the melanoma patients. While $30.6 \%$ of the samples without ulceration stained positive for nuclear DROSHA, only $6.9 \%$ of those with ulceration had positive nuclear DROSHA staining $\left(P=0.002, \quad \chi^{2}\right.$ test; Table 1). In addition, when compared with the nuclear DROSHA staining between different subtypes of melanoma, we found that lentigo maligna and superficial spreading subtypes express less DROSHA than other subtypes $(P=0.016$; Table 1$)$.

Importantly, our data also demonstrated that nuclear DROSHA expression is inversely correlated with American Joint Committee on Cancer (AJCC) stages of melanoma $\left(P=0.0001, \chi^{2}\right.$ test; Figure $\left.2 b\right)$. We found that the main and only significant difference in nuclear DROSHA staining exist between stage I and II $\left(P=0.0001, \chi^{2}\right.$ test $)$ but not the other stages $\left(P>0.05, \chi^{2}\right.$ test $)$.

\section{Subcellular Compartment Shift of DROSHA Expression to the Cytoplasm during Melanoma Progression}

As mentioned earlier, in addition to the nuclear staining for DROSHA we also observed cytoplasmic staining for this protein in all stages of melanocytic lesion. To investigate if the cytoplasmic nuclear staining has any significance in melanoma progression, we evaluated DROSHA staining in cytoplasm. Kruskal-Wallis test revealed a marked increase in cytoplasmic expression of DROSHA during melanoma progression $(P=0.026$; Figure 3a). Significant difference for the cytoplasmic DROSHA staining was observed between dysplastic nevi and primary melanomas $(P=0.016)$, but not between normal nevi and dysplastic nevi or between primary melanomas and metastatic melanomas $(P>0.05)$. Furthermore, to examine whether the reduced nuclear DROSHA staining is due to a subcellular shift, we divided the samples in each stage of melanoma progression into four categories based on their nuclear and cytoplasmic DROSHA expression profile: (1) negative nuclei and cytoplasm; (2) negative nuclei but positive cytoplasm; (3) positive nuclei but negative cytoplasm; (4) positive nuclei and cytoplasm. Interestingly, we observed a considerable shift in DROSHA expression from nuclei to cytoplasm during melanoma progression $\left(P=0.0001, \chi^{2}\right.$ test; Figure $\left.3 \mathrm{~b}\right)$.

Table 1 Nuclear Drosha staining and clinicopathologic characteristics of 226 primary melanomas

\begin{tabular}{|c|c|c|c|c|}
\hline \multirow[b]{2}{*}{ Variables } & \multicolumn{3}{|c|}{ Nuclear DROSHA staining } & \multirow[b]{2}{*}{$\mathrm{P}$ value ${ }^{a}$} \\
\hline & Negative & Positive & Total & \\
\hline \multicolumn{5}{|l|}{ Age (years) } \\
\hline$\leq 63$ & $81(71.1 \%)$ & $33(28.9 \%)$ & 114 & 0.327 \\
\hline$>63$ & $86(76.8 \%)$ & $26(23.2 \%)$ & 112 & \\
\hline \multicolumn{5}{|l|}{ Sex } \\
\hline Male & $91(71.6 \%)$ & $36(28.4 \%)$ & 127 & 0.385 \\
\hline Female & $76(76.8 \%)$ & $23(23.2 \%)$ & 99 & \\
\hline \multicolumn{5}{|l|}{ Ulceration } \\
\hline Present & $40(93.0 \%)$ & $3(7.0 \%)$ & 43 & 0.002 \\
\hline Absent & $127(69.4 \%)$ & $56(30.6 \%)$ & 183 & \\
\hline \multicolumn{5}{|l|}{ Lymphocytic response } \\
\hline Present & $56(75.7 \%)$ & $18(24.3 \%)$ & 74 & 0.670 \\
\hline Absent & $111(73.0 \%)$ & $41(27.0 \%)$ & 152 & \\
\hline \multicolumn{5}{|l|}{ Tumor subtype } \\
\hline Lentigo maligna & $36(65.5 \%)$ & $19(34.5 \%)$ & 55 & 0.016 \\
\hline Superficial spreading & $56(68.3 \%)$ & $26(31.7 \%)$ & 82 & \\
\hline Other ${ }^{b}$ & $75(84.3 \%)$ & $14(15.7 \%)$ & 89 & \\
\hline \multicolumn{5}{|l|}{ Site $^{\mathrm{c}}$} \\
\hline Sun-exposed & $44(74.6 \%)$ & $15(25.4 \%)$ & 59 & 0.890 \\
\hline Sun-protected & $123(73.7 \%)$ & $44(26.3 \%)$ & 127 & \\
\hline
\end{tabular}

$\mathrm{a}^{2}$ test.

b ${ }^{\chi}$ Other: unspecified subtype.

${ }^{\mathrm{C}}$ Sun-protected sites: trunk, arm, leg and feet; sun-exposed sites: head and neck. 

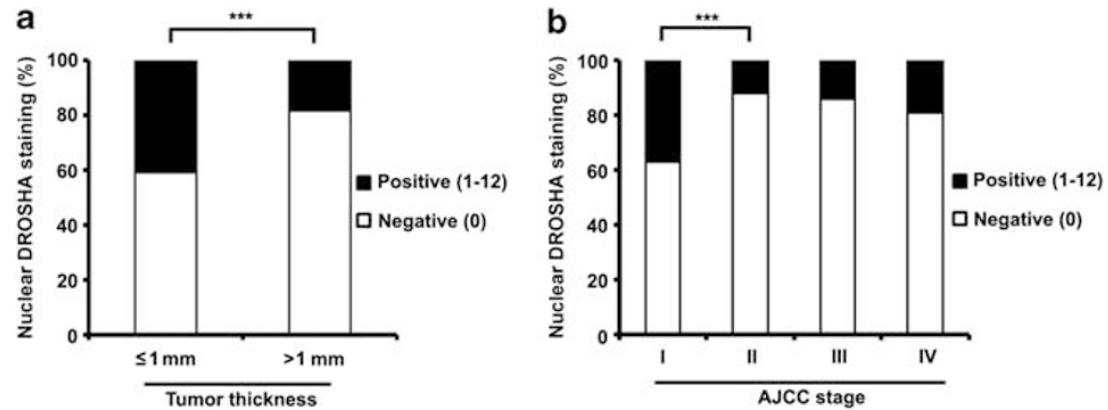

Figure 2 Nuclear Drosha expression is negatively correlated with (a) tumor thickness $\left(P=0.0001, \chi^{2}\right.$ test), and (b) AJCC stage of melanoma $\left(P=0.0001, \chi^{2}\right.$ test $)$.
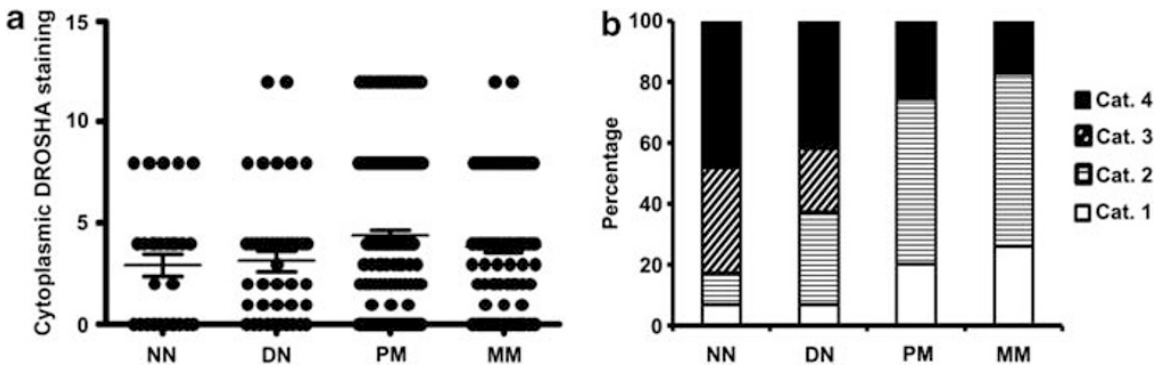

Figure 3 Cytoplasmic expression of Drosha is increased during melanoma progression. (a) Kruskal-Wallis test for differences in cytoplasmic expression of Drosha among NN, DN, PM, and MM $(P=0.026)$. Significant difference was found between dysplastic nevi and primary melanomas $(P=0.016)$, but not between normal nevi and dysplastic nevi or between primary melanomas and metastatic melanomas $(P>0.05)$. (b) Combined analysis of nuclear and cytoplasmic Drosha staining in NN, DN, PM, and MM. Each sample was categorized based on nuclear and cytoplasmic Drosha expression as following: (1) negative nuclei and negative cytoplasm; (2) negative nuclei but positive cytoplasm; (3) positive nuclei but negative cytoplasm; and (4) positive nuclei and positive cytoplasm.

Accordingly, category 2 that represents samples with negative nuclear but positive cytoplasmic staining for DROSHA increased from $10.3 \%$ in normal nevi to $30.2 \%$ in dysplastic nevi, $54.2 \%$ in primary melanomas and $56.8 \%$ in metastatic melanomas, whereas category 3 that represents samples with positive nuclear but negative cytoplasmic staining for DROSHA decreased from $34.5 \%$ in normal nevi to $20.9 \%$ in dysplastic nevi, and further to $1.3 \%$ in primary melanomas and $0.9 \%$ in metastatic melanomas.

\section{Validation of DROSHA Staining with a Second Antibody}

To further validate our observation that expression of nuclear DROSHA is reduced in melanoma, we used a second rabbit polyclonal antibody against the C-terminal (amino acids 1071-1370) to probe for expression of DROSHA in a smaller TMA construct, containing 48 dysplastic nevi, 86 primary melanomas, and 49 metastatic melanomas (Figure 4). We observed an expression pattern of nuclear DROSHA very similar to the first antibody by using this antibody (Figure 4a). Indeed, Kruskal-Wallis test revealed a reduced expression of nuclear DROSHA during melanoma progression $(P=0.0001$; Figure 4b). Furthermore, when we divided the samples from each stage into two groups (positive and negative) based on expression of nuclear DROSHA, we observed an increase in percentage of samples with no nuclear DROSHA staining during melanoma progression $\left(P=0.001, \chi^{2}\right.$ test; Figure 4c). Accordingly, while $56.2 \%$ of dysplastic nevi had positive nuclear staining for DROSHA, only $20.9 \%$ of primary melanomas and $12.2 \%$ of metastatic melanomas stained positive for nuclear DROSHA.

Concomitant Loss of DROSHA and DICER1 Expression Renders Worse Prognosis for Melanoma Progression

To evaluate whether the reduced nuclear DROSHA staining in human melanomas correlates with patient survival, we constructed Kaplan-Meier survival curves using overall or disease-specific 5 -year survival to compare biopsies with positive nuclear DROSHA staining to those with negative nuclear DROSHA staining. Our results revealed that despite a trend toward higher survival rate in samples with positive nuclear DROSHA signals, this correlation is not significant for either overall $(P=0.073)$ or disease-specific $(P=0.097)$ 5-year survival of the patients (data not shown). Our analysis with the second anti-DROSHA antibody 
a

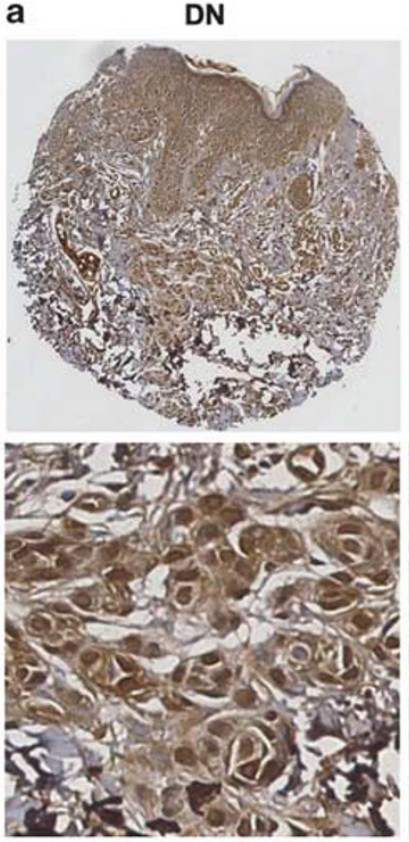

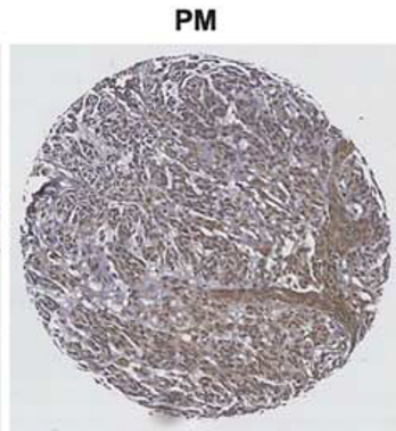
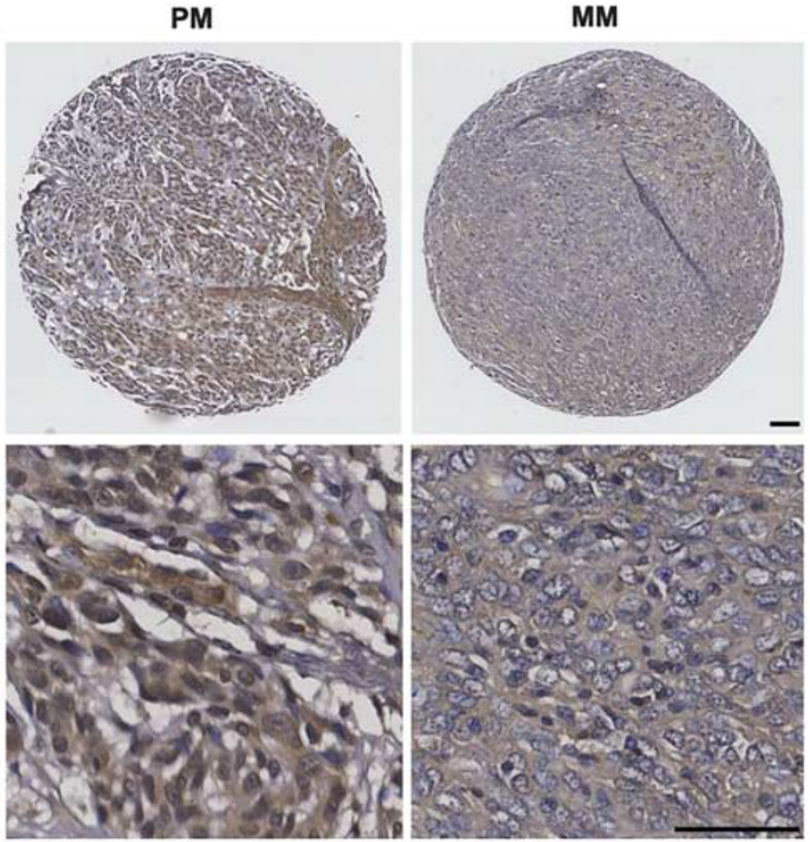

b

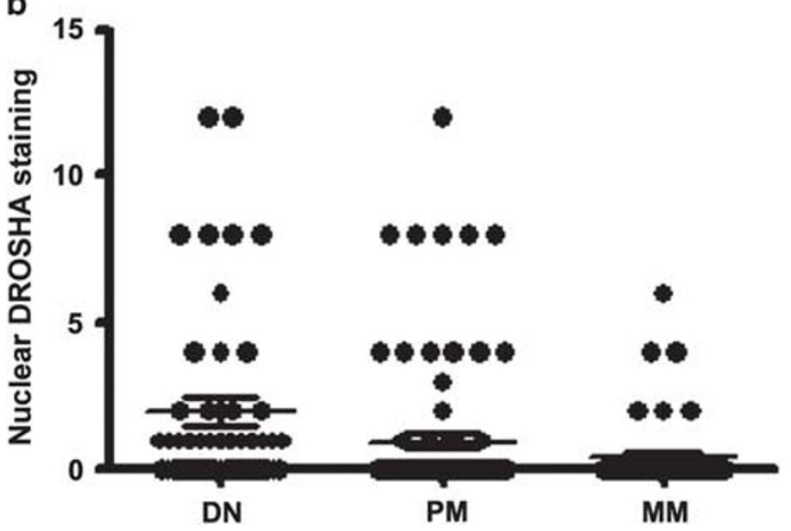

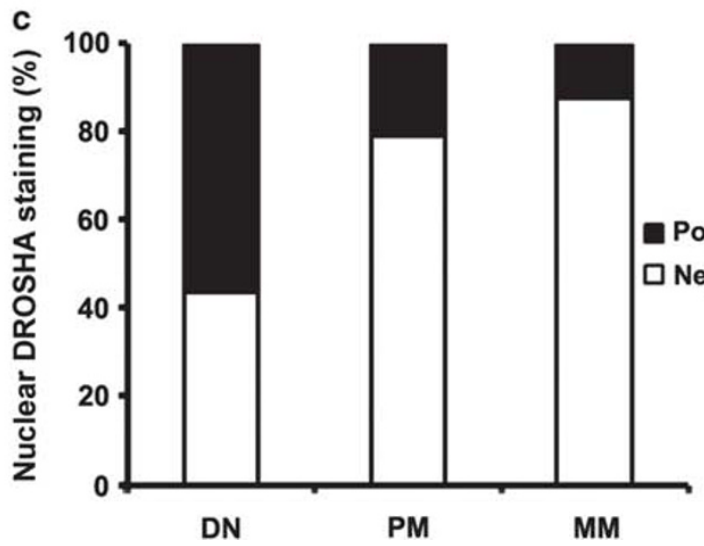

Figure 4 Tissue microarray using a second specific antibody raised against the C-terminal region of the human Drosha protein confirms the reduced nuclear Drosha expression during melanoma progression. (a) Representative images of dysplastic nevi (DN) with strong nuclear Drosha staining, primary melanoma (PM) with weak and metastatic melanoma (MM) with negative nuclear Drosha staining. (b) Kruskal-Wallis test for differences in nuclear Drosha staining among DN, PM, and MM. The median is depicted as a horizontal line in each group $(P=0.0001)$. Significant difference was found between DN and PM $(P=0.001$, Mann-Whitney test $)$ but not PM and MM $\left(P=0.36\right.$, Mann-Whitney test). (c) $\chi^{2}$ test for differences in nuclear Drosha staining in DN, PM, and MM. Significant difference was found between DN and PM $(P=0.001)$ but not PM and MM $(P=0.204)$.

also produced similar non-significant results (data not shown).

We previously reported that expression of cytoplasmic DICER1 is reduced during melanoma progression towards metastasis. ${ }^{19}$ Since DROSHA and DICER1 are two main players in the miRNA biogenesis pathway, we investigated the potential correlation in the expression of these two proteins in melanocytic lesions and the correlation between concomitant expression of these two factors and melanoma patients' survival. We found that despite reduced expression of cytoplasmic DICER1 and nuclear DROSHA during melanoma progression, there is no significant correlation between their expression patterns in the single sample level (data not shown). However, when we divided the samples into three groups; positive DROSHApositive DICER1 (category 1; $n=49$ ), positive DICER1-negative DROSHA or negative DICER1positive DROSHA (category 2; $n=192$ ) and negative DICER1-negative DROSHA (category 3; $n=33$ ), we found a significant correlation between their concomitant expression and patient survival $(P=0.019$ for overall and 0.001 for disease-specific survival; Figure 5a and b). We observed that samples in category 3 represented the worst survival outcome $(36.7 \%$ for both overall and disease-specific survival; Figure $5 \mathrm{a}$ and b), whereas category 1 represented the best outcome $(65.3 \%$ for overall and $75.5 \%$ for disease-specific survival; Figure 5a 

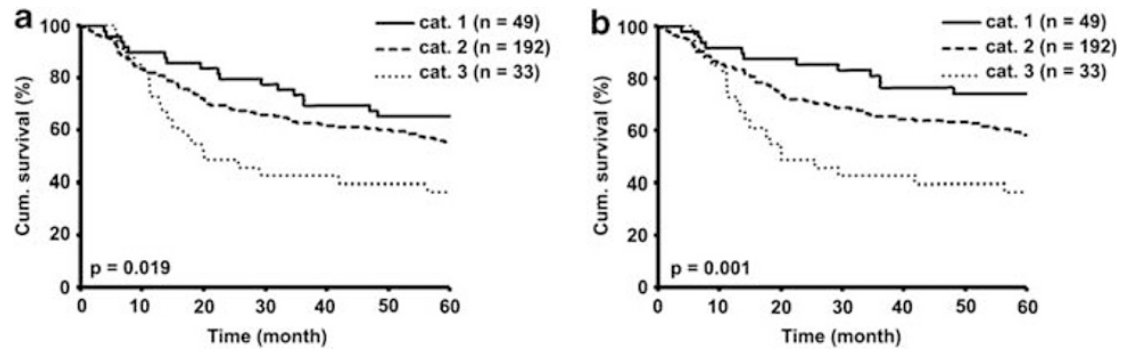

Figure 5 Concomitant loss of nuclear Drosha and cytoplasmic DICER1 inversely correlates with (a) overall and (b) disease-specific 5-year survival of all primary and metastatic melanoma patients.

and b). Interestingly, survival rate for samples in category 2 was between the other two categories $(54.7 \%$ for overall and $59.4 \%$ for disease-specific survival; Figure 5a and b).

\section{Discussion}

Expression pattern of DROSHA has been investigated in regard to tumor progression and survival prediction in a number of malignancies. However, to our knowledge, this is the first study to investigate the DROSHA protein expressions in human cutaneous melanoma. Using two different antibodies raised against different regions of the DROSHA protein, we observed a marked downregulation of nuclear DROSHA expression during melanoma progression (Figures 1 and 4). We also observed an inverse correlation between nuclear expression of DROSHA and tumor thickness, AJCC stage, and ulceration status (Figure 2; Table 1). These data indicate a possible tumor suppressor function for DROSHA in melanoma.

Interestingly, we also observed an increased cytoplasmic staining for DROSHA along with the reduced nuclear DROSHA expression during melanoma progression (Figure 3). This observation suggests that the nuclear-to-cytoplasm translocation of DROSHA protein may be a relevant event in melanomagenesis. In esophageal cancer samples, DROSHA was shown by immunohistochemistry to be strongly expressed in both nuclei and cytoplasm. ${ }^{23}$ Cytoplasmic localization of DROSHA was also recently reported in colorectal carcinoma cells by immunofluorescence analyses. ${ }^{24}$ Similarly, cytoplasmic DROSHA expression was detected in MCF-7 breast cancer cell line. ${ }^{25}$

It should be noted that alternatively spliced transcripts, encoding C-terminally truncated DROSHA proteins lacking part of the RIIIDb and the entire dsRBD have been detected in human melanoma cell lines. Proteins generated from these alternative splice variants fail to bind to DGCR8 and form the microprocessor complex. ${ }^{26}$ These splice variants are deficient in pri-miRNA processing. However, these aberrant transcripts in melanoma cells do not hamper miRNA biogenesis process. ${ }^{26} \mathrm{In}$ our study, it is possible that these splice isoforms contribute to the signals detected by the antibody that targets the N-terminal of DROSHA protein; nevertheless, we believe that this possibility is remote since the second antibody that targets the C-terminal of the DROSHA protein (therefore, unable to detect these isoforms) shows a pattern of DROSHA expression in melanocytic lesions very similar to the other antibody.

Previous reports demonstrated various degrees of correlation between DROSHA expression and survival $^{13,20,22-24,27,28}$ Nevertheless, despite a clear trend, our Kaplan-Meier analysis did not reveal a significant correlation between nuclear DROSHA expression and patients' overall or disease-specific survival. This lack of significant correlation may be explained by the notion that reduction of nuclear DROSHA expression mainly happens at earlier stages of melanomagenesis (from normal nevi to dysplastic nevi and further from dysplastic nevi to primary melanomas; Figures 1 and 4).

A previous report on breast cancers showed that DROSHA and DICER1 were concurrently downregulated in $15 \%$ of cases. $^{28}$ Downregulation of DROSHA in endometrial cancer was also shown to be significantly correlated with decreased expression of DICER1. ${ }^{29}$ Nonetheless, we did not observe any correlation between nuclear DROSHA and cytoplasmic DICER1 expression in melanocytic lesions. In addition, when we compared the expression pattern of nuclear DROSHA with that of cytoplasmic DICER1 during melanoma progression, ${ }^{19}$ we observed that while the expression of DICER1 reduces mainly in the latest stage of melanoma progression (metastatic melanoma), nuclear DROSHA expression is progressively reduced in almost all stages of melanoma progression. These data indicate that expression pattern of these two factors differ in different stages of melanoma progression, suggesting the existence of distinct mechanisms responsible for differential regulation of their expression. However, despite this lack of correlation at the expression level, our survival analysis revealed that concomitant expression of these two factors confers the best survival outcome for the patients (Figure 4). In line with our observation in melanoma, studies in ovarian cancer showed that cases with both high DICER1 expression and high DROSHA expression 
were associated with increased survival. ${ }^{13}$ Altogether, our data suggest that integrity of the miRNA biogenesis pathway is perturbed in a considerable subset of melanomas and that those cases whose expression pattern of miRNA biogenesis factors (and probably expression pattern of miRNAs) resembles that of normal tissues represent a better rate of survival.

Loss of expression of DROSHA and DICER1 has been shown before to cause different phenotypes. For instance, it was reported that DICER1 knockdown significantly decreases migration of endothelial cells, whereas DROSHA knockdown has no effect. ${ }^{30}$ Similarly, silencing of DICER1 but not DROSHA reduces angiogenesis in vivo. ${ }^{30}$ Also, Chong et $a l^{31}$ revealed that in early stage thymocytes, deficiency in DROSHA or DICER1 does not always result in identical phenotypes and using the mouse embryonic fibroblasts they showed that loss of DROSHA expression results in a pattern of gene expression different than loss of DICER1. Interestingly, they showed that DROSHA also recognizes and directly cleaves many mRNAs with secondary stem-loop structures. In addition, a subset of miRNAs was found to be generated by a DICER1-dependent but DROSHA-independent mechanism, explaining the distinct and non-overlapping phenotypes caused by loss of DROSHA and DICER1. ${ }^{31}$ Reduced DROSHA, but not DICER1, expression in human mesenchymal stem cells was demonstrated to significantly reduce proliferation rate. $^{32}$ In addition, unlike DICER1 knockdown, DROSHA knockdown human mesenchymal stem cells contained an increased number of G1 phase cells, with a reduced level of cells in $S$ phase, accompanied by decreased pRB, $28 \mathrm{~S}$ and $18 \mathrm{~S}$ rRNA expression and increased p16 and p15 (two key regulators of the G1/S phase transition) expression, indicating that DROSHA modifies human mesenchymal stem cells proliferation through a miRNAindependent mechanism. ${ }^{32}$ Our data demonstrating further reduction in survival rate of the melanoma cases with negative DICER1-negative and DROSHA expression compared with that of the cases with loss of either DICER1 or DROSHA (Figure 5) also indicate the possible existence of at least some non-overlapping functions of these two factors. However, beside its direct role in biogenesis of miRNAs, so far very little is known of putative functions of DROSHA in melanocytic cells. Future studies will delineate these functions of DROSHA and its possible contribution to suppression of melanomagenesis.

In conclusion, our data revealed a decreased nuclear expression of DROSHA during melanoma progression along with subcellular compartment shift of its expression toward the cytoplasm. We observed that expression of DROSHA inversely correlates with tumor thickness, AJCC stage and ulceration status. In addition, we revealed that concurrent loss of expression of DROSHA and
DICER1 confers an adverse affect on melanoma patient survival, worse than singular loss of each of these factors. Our data suggest a possible tumor suppressor function for DROSHA in melanoma and highlights the requirement for future studies on its role in this type of malignancy and it possible miRNA-independent functions.

\section{Acknowledgements}

We thank Dr Gholamreza Safaee Ardekani, Dr Ladan Fazli and Scot Kwong for technical assistance and Drs Michael Cox, Aziz Ghahary and Vincent Duronio for helpful discussions. This work was funded by grants from the Canadian Institutes of Health Research (MOP-93810, MOP-110974 and CCI-117958) and Canadian Dermatology Foundation to GL. SMJ is a recipient of Sandy Kyo-Hyun Park Scholarship in Cancer Research, University of British Columbia Graduate Fellowship and Canadian Institutes of Health Research-Skin Research Training Centre Trainee Awards.

\section{Disclosure/conflict of interest}

The authors declare no conflict of interest.

\section{References}

1 Bushati N, Cohen SM. microRNA functions. Annu Rev Cell Dev Biol 2007;23:175-205.

2 Krol J, Loedige I, Filipowicz W. The widespread regulation of microRNA biogenesis, function and decay. Nat Rev Genet 2010;11:597-610.

3 Carrington JC, Ambros V. Role of microRNAs in plant and animal development. Science 2003;301:336-338.

4 Cheng AM, Byrom MW, Shelton J, et al. Antisense inhibition of human miRNAs and indications for an involvement of miRNA in cell growth and apoptosis. Nucleic Acids Res 2005;33:1290-1297.

5 Chen Y, Gorski DH. Regulation of angiogenesis through a microRNA (miR-130a) that down-regulates antiangiogenic homeobox genes GAX and HOXA5. Blood 2008;111:1217-1226.

6 Wilting SM, Snijders PJ, Verlaat W, et al. Altered microRNA expression associated with chromosomal changes contributes to cervical carcinogenesis. Oncogene 2013;32:106-116.

7 Landi MT, Zhao Y, Rotunno M, et al. MicroRNA expression differentiates histology and predicts survival of lung cancer. Clin Cancer Res 2010;16:430-441.

8 Ozen M, Creighton CJ, Ozdemir M, et al. Widespread deregulation of microRNA expression in human prostate cancer. Oncogene 2008;27:1788-1793.

9 Png KJ, Yoshida M, Zhang XHF, et al. MicroRNA-335 inhibits tumor reinitiation and is silenced through genetic and epigenetic mechanisms in human breast cancer. Gene Dev 2011;25:226-231.

10 Baer C, Claus R, Frenzel LP, et al. Extensive promoter hypermethylation and hypomethylation is associated with aberrant microRNA expression in 
chronic lymphocytic leukemia. Cancer Res 2012;72: 3775-3785.

11 Kuchen S, Resch W, Yamane A, et al. Regulation of microRNA expression and abundance during lymphopoiesis. Immunity 2010;32:828-839.

12 Faggad A, Kasajima A, Weichert W, et al. Downregulation of the microRNA processing enzyme Dicer is a prognostic factor in human colorectal cancer. Histopathology 2012; in press.

13 Merritt WM, Lin YG, Han LY, et al. Dicer, Drosha, and outcomes in patients with ovarian cancer. N Engl J Med 2008;359:2641-2650.

14 Zhou YM, Chen LJ, Barlogie B, et al. High-risk myeloma is associated with global elevation of miRNAs and overexpression of EIF2C2/AGO2. Proc Natl Acad Sci USA 2010;107:7904-7909.

15 Caramuta S, Egyhazi S, Rodolfo M, et al. MicroRNA expression profiles associated with mutational status and survival in malignant melanoma. J Invest Dermatol 2010;130:2062-2070.

$16 \mathrm{Xu}$ Y, Brenn T, Brown ER, et al. Differential expression of microRNAs during melanoma progression: miR-200c, miR-205 and miR-211 are downregulated in melanoma and act as tumour suppressors. Br J Cancer 2012;106:553-561.

17 Schultz J, Lorenz P, Gross G, et al. MicroRNA let-7b targets important cell cycle molecules in malignant melanoma cells and interferes with anchorageindependent growth. Cell Res 2008;18:549-557.

18 Felicetti F, Errico MC, Bottero L, et al. The promyelocytic leukemia zinc finger-microRNA-221/-222 pathway controls melanoma progression through multiple oncogenic mechanisms. Cancer Res 2008;68: 2745-2754.

19 Jafarnejad SM, Ardekani GS, Ghaffari M, et al. Sox4-mediated Dicer expression is critical for suppression of melanoma cell invasion. Oncogene 2012; in press.

20 Guo XF, Liao QJ, Chen $\mathrm{P}$, et al. The microRNAprocessing enzymes: Drosha and Dicer can predict prognosis of nasopharyngeal carcinoma. J Cancer Res Clin 2012;138:49-56.

21 Papachristou DJ, Sklirou E, Corradi D, et al. Immunohistochemical analysis of the endoribonucleases
Drosha, Dicer and Ago2 in smooth muscle tumours of soft tissues. Histopathology 2012;60:E28-E36.

22 Shu GS, Yang ZL, Liu DC. Immunohistochemical study of Dicer and Drosha expression in the benign and malignant lesions of gallbladder and their clinicopathological significances. Pathol Res Pract 2012;208:392-397.

23 Sugito N, Ishiguro H, Kuwabara Y, et al. RNASEN regulates cell proliferation and affects survival in esophageal cancer patients. Clin Cancer Res 2006;12: 7322-7328.

24 Papachristou DJ, Korpetinou A, Giannopoulou E, et al. Expression of the ribonucleases Drosha, Dicer, and Ago2 in colorectal carcinomas. Virchows Arch 2011;459:431-440.

25 Passon N, Gerometta A, Puppin C, et al. Expression of Dicer and Drosha in triple-negative breast cancer. J Clin Pathol 2012;65:320-326.

26 Grund SE, Polycarpou-Schwarz M, Luo C, et al. Rare Drosha splice variants are deficient in microRNA processing but do not affect general microRNA expression in cancer cells. Neoplasia 2012;14:238-248.

27 Vaksman O, Hetland TE, Trope CG, et al. Argonaute, Dicer, and Drosha are up-regulated along tumor progression in serous ovarian carcinoma. Hum Pathol 2012;43:2062-2069.

28 Dedes KJ, Natrajan R, Lambros $\mathrm{MB}$, et al. Down-regulation of the miRNA master regulators Drosha and Dicer is associated with specific subgroups of breast cancer. Eur J Cancer 2011;47:138-150.

29 Torres A, Torres K, Paszkowski T, et al. Major regulators of microRNAs biogenesis Dicer and Drosha are down-regulated in endometrial cancer. Tumour Biol 2011;32:769-776.

30 Kuehbacher A, Urbich C, Zeiher AM, et al. Role of dicer and Drosha for endothelial microRNA expression and angiogenesis. Circ Res 2007;101:59-68.

31 Chong MM, Zhang G, Cheloufi S, et al. Canonical and alternate functions of the microRNA biogenesis machinery. Genes Dev 2010;24:1951-1960.

32 Oskowitz AZ, Penfornis P, Tucker A, et al. Drosha regulates hMSCs cell cycle progression through a miRNA independent mechanism. Int J Biochem Cell B 2011:43:1563-1572. 